\title{
Double Optical Tweezers for 3D Photonic Force Measurements
}

\author{
A. A. R. Neves, A. Fontes, W. L. Moreira, A. A. de Thomaz, D. B. Almeida, L. C. Barbosa and C. L. \\ Cesar.
}

Instituto de Física Gleb Wataghin - Universidade Estadual de Campinas - 13083-970 - Campinas SP - Brazil.

Optical tweezers have become an important tool for biological manipulations and cell mechanical properties measurements [1]. These measurements use the displacement from equilibrium position of a microsphere as the force transducer. Therefore, the calibration procedure requires the use of good models for the optical force in microspheres. Geometrical optics has been used when the particle dimensions are much greater than the light wavelength, and Rayleigh scattering theory for the opposite. However, when the particles are of the same order of the wavelength these approximations are no longer valid and full vectorial electromagnetic theory is necessary. Mie resonances are typical of this size regime. Moreover, just the description of a vectorial electromagnetic field of a very high numerical aperture beam is still an open field [2,3]. Therefore good sensitive optical force measurements are necessary to discriminate among the several models. In previous work we have shown that the double optical tweezers can be used to perform an ultrasensitive force spectroscopy by observing forces due to the light scattering and selectively coupling the light to either the TE, the TM or both TE and TM microsphere modes in a single isolated particle as function of the beam polarization and position [4]. Our results showed how careful one has to be when using optical force models for mechanical properties measurements. The microsphere modes can change the force values by more than $30-50 \%$. Also it clearly shows how the usually assumed azimuthal symmetry in the horizontal plane no longer holds because the beam polarization breaks this symmetry. In this article we continue the investigation of the forces in an optical tweezers by using this ultrasensitive experimental technique to measure whole optical force curves as a function of the 3D beam position and polarization. The importance of understanding the optical scattering forces of dielectric microspheres under different incident beam conditions comes from the fact that they have been used as the natural transducer for force and other mechanical measurements. Because optical tweezers itself is an ultrasensitive technique, we used another optical tweezers to characterize the optical force of microspheres.

For the experimental setup used, the beam from a Nd:YAG cw laser (denoted as trapping beam) is used to keep the particle trapped while the beam from a tunable Ti:Sapphire cw laser, which is modulated and highly attenuated (denoted as perturbing beam), is used to perturb the particle from its equilibrium position. Some advantages of this setup are that it allows: 1. the measurement to be performed in suspended and isolated particles trapped for hours; 2 . to observe the same particle submitted to different conditions; 3 . to localize the perturbing beam in any desired position at the sphere. A signal proportional to the displacement of the bead was measured using the back-scattering of a HeNe laser. The axial and radial forces were observed by monitoring the amplitude of the displacements while changing the position of the Ti:Sapphire laser. The translation of the focal spot in the focal plane of the objective is accomplished by the use of a gimbal mount. The distance between the laser spot on the mirror and the objective pupil is constant, in conjugate planes, so a change in beam angle at the mirror corresponds to a change of angle at the objective. The axial control of the focal spot in the objective focal plane is obtained adjusting the beam divergence without changing the diameter at the objective aperture. This is accomplished by constraining the 
beam diameter at the gimbal mirror independent of the changes in beam divergence. With these two techniques our setup is capable of a full 3D translation of our laser spot inside of a Neubaur chamber. The rotation and translation are computer controlled by a step motor. To relate the motor steps to microns a previous calibration run was executed. Measurements to characterize the optical force as a function of polarization were done in the radial and axial position for $3 \mu \mathrm{m}, 6 \mu \mathrm{m}$ and 9 $\mu \mathrm{m}$ polystyrene spheres diluted in water and placed in a Neubauer chamber. Some of the measurements are shown in figure $1(a, b)$.

Classical Mie scattering theory was developed for plane waves and cannot explain the measurements obtained by this focused beam experiment. In this case, it is necessary to decompose the incident beam in plane waves relative to the center of the microsphere. As the beam focus is no longer at the origin of the coordinate system all the beam azimuthal symmetry is lost. This can be a complicated problem, especially when a full vectorial diffraction description of the electromagnetic fields and highly focused laser beams are required. All sorts of approximations and tricks have been used to proceed forward to obtain numerical results $[2,3]$. Although this is an old subject included in text books on Electrodynamics [5], where they express the beam shape coefficients multiplied by a spherical Bessel function of argument $\mathrm{kr}$ in terms of an integration over the solid angle, they fail to prove that the solid angle integration will generate the same spherical Bessel function of the left hand side of the equation. We used this our theory for exact partial wave expansion of optical beams with respect to arbitrary origin [6] to explain the results obtained with this ultrasensitive experimental technique described above.
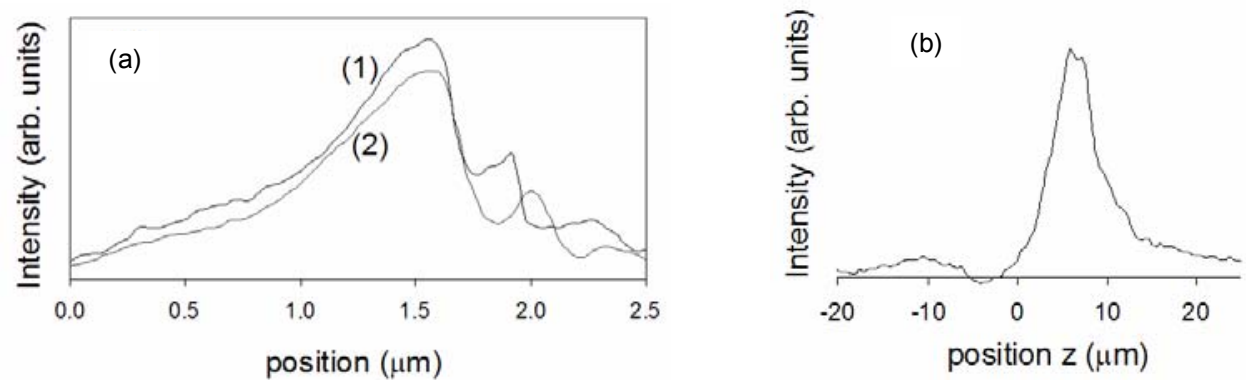

Fig1. (a) Experimental for the radial force for a $3 \mu \mathrm{m}$ sphere moving (1) perpendicular to the polarization and (2) parallel to the polarization. (b) Experimental data for the axial force in $6 \mu \mathrm{m}$ sphere.

[1]. A. Ashkin, J. M. Dziedzic, J. E. Bjorkholm and S. Chu, Opt. Lett. 11 (1986) 288.

[2] J. A. Lock, Appl. Opt. 43 (2004) 2532.

[3] A. Mazolli, P. A. M. Neto, H. M Nussenzveig, Proc. Royal Soc. London Ser. A Math. Phys. Eng. Sci. 459 (2003) 3021.

[4]. A. Fontes, A. A. R. Neves, W. L. Moreira, A. A. de Thomaz, L. C. Barbosa, A. M. De Paula, C. L. Cesar, Appl. Phys. Lett. 87 (2005) Art. No. 221109.

[5]. C. F. Bohren and D. R. Huffman in Absorption and Scattering of Light by Small Particles, John Wiley \& Sons, New York, 1983.

[6]. A. A. R. Neves, A. Fontes, L. A. Padilha, E. Rodriguez, C. H. Brito Cruz, L. C. Barbosa, C. L. Cesar, submitted to Phys. Rev. Lett. (2006). 